\title{
A single case of MRI-guided focused ultrasound ventro-oral thalamotomy for musician's dystonia
}

\author{
Shiro Horisawa, MD, ${ }^{1}$ Toshio Yamaguchi, MD, PhD, ${ }^{2}$ Keiichi Abe, MD, ${ }^{1}$ Hiroki Hori, DC, ${ }^{3}$ \\ Masatake Sumi, MD, ${ }^{1}$ Yoshiyuki Konishi, PhD, ${ }^{3}$ and Takaomi Taira, MD, PhD ${ }^{1}$
}

\begin{abstract}
'Department of Neurosurgery, Tokyo Women's Medical University, Tokyo; ²Department of Radiology, Shin-Yurigaoka General Hospital, Kawasaki; and ${ }^{3}$ Faculty of Advanced Techno-Surgery, Institute of Advanced Biomedical Engineering \& Science, Graduate School of Medicine, Tokyo Women's Medical University, Tokyo, Japan
\end{abstract}

\begin{abstract}
Musician's dystonia (MD) is a type of focal hand dystonia that develops only while playing musical instruments and interferes with skilled and fine movements. Lesioning of the ventro-oral (Vo) nucleus of the thalamus (Vo-thalamotomy) using radiofrequency can cause dramatic improvement in MD symptoms. Focused ultrasound (FUS) can make intracranial focal lesions without an incision. The authors used MRI-guided FUS (MRgFUS) to create a lesion on the Vo nucleus to treat a patient with MD. Tubiana's MD scale (TMDS) was used to evaluate the condition of musical play ranging from 1 to 5 (1: worst, 5 : best). The patient was a 35-year-old right-handed man with involuntary flexion of the right second, third, and fourth fingers, which occurred while playing a classical guitar. Immediately after therapeutic sonications of FUS Vothalamotomy, there was dramatic improvement in the MD symptoms. The TMDS scores before; at 0 and 1 week after; and at $1,3,6$, and 12 months after MRgFUS Vo-thalamotomy were 1, 4, 4, 5, 5, 5, and 5, respectively. No complications were observed. Focused ultrasound Vo-thalamotomy can be an effective treatment for MD.
\end{abstract}

https://thejns.org/doi/abs/10.3171/2018.5.JNS173125

KEYWORDS focused ultrasound; thalamotomy; ventro-oral nucleus; dystonia; functional neurosurgery

$\mathrm{D}$ YSTONIA is a movement disorder characterized by involuntary, sustained, and patterned muscle contractions. ${ }^{4}$ Focal dystonia affects one part of the body and includes blepharospasm, cervical dystonia, focal hand dystonia, and other dystonias. Musician's dystonia (MD) is a type of focal dystonia, mainly involving focal hand dystonia, which develops only while playing musical instruments and interferes with skilled and fine movements. ${ }^{17}$ Musician's dystonia is more common among musicians in their 30s after years of repetitive and fine movements associated with playing musical instruments. ${ }^{9}$ Approximately $1 \%$ of professional musicians are affected with MD during their career. ${ }^{1}$ Various treatments for MD are available, including botulinum toxin injections, oral medications, and physical therapy.1,14,18 Despite these conservative treatments, more than $50 \%$ of affected musicians ultimately give up their professional musical career. ${ }^{15}$ For patients with MD refractory to conservative treatments, lesioning of the ventro-oral ( $\mathrm{Vo}$ ) nucleus of the thalamus (Vo-thalamotomy) can cause dramatic improvement of symptoms. ${ }^{7}$ Recently, MRI-guided focused ultrasound
(MRgFUS) thalamotomy, which allows intracranial focal lesioning without an incision, at the ventral intermediate thalamic nucleus (VIM) has been reported to be a safe, effective, and feasible procedure for essential tremor. ${ }^{3}$ Previously, we reported the effectiveness of Vo-thalamotomy using radiofrequency and Gamma Knife ablation for MD. ${ }^{7,8}$ Here, we report the effectiveness of MRgFUS Vothalamotomy for MD. To our knowledge, this is the first case report of MRgFUS Vo-thalamotomy for MD with a successful outcome at the 1-year follow-up.

\section{Case Report}

History and Examination

The patient was a 35-year-old right-handed man with involuntary flexion of the right second, third, and fourth fingers, which occurred while playing a classical guitar. He had started playing guitar in his teens. At the age of 26 years, involuntary flexion of the right third finger developed only while playing a classical guitar. Subsequently, involuntary flexion of the right second and fourth fingers

ABBREVIATIONS FUS = focused ultrasound; GKT = Gamma Knife thalamotomy; MD = musician's dystonia; MRgFUS = MRI-guided FUS; TMDS = Tubiana's MD scale; $V_{0}=$ ventro-oral; Vo-thalamotomy = lesioning of the Vo nucleus of the thalamus.

SUBMITTED December 13, 2017. ACCEPTED May 10, 2018.

INCLUDE WHEN CITING Published online September 21, 2018; DOI: 10.3171/2018.5.JNS173125. 

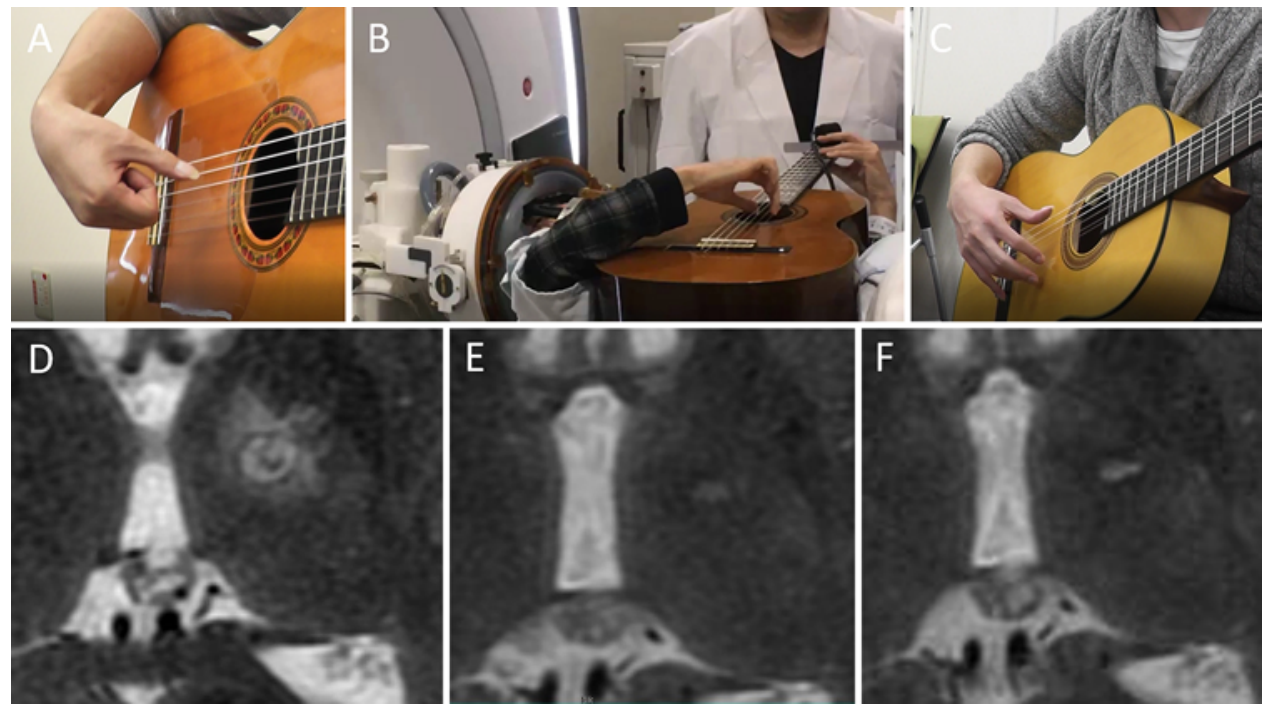

FIG. 1. Pre- and postoperative neurological conditions and MRI studies. Involuntary flexion of the right second, third, and fourth fingers while playing a guitar (A). Fluctuation in symptoms was carefully assessed with an MRI-compatible guitar (B). One week after MRgFUS Vo-thalamotomy, improvement in MD symptoms was seen (C). Coronal T2-weighted MR image at 0 (D), 3 (E), and 12 (F) months after MRgFUS Vo-thalamotomy. The lesion at 12 months is still visible. Figure is available in color online only.

developed at the ages of 28 and 33 years, respectively (Fig. 1A). Since the age of 29 years, the symptoms also manifested while using a keyboard and while writing. The patient underwent tendon elongation of the affected fingers, which showed no effect. He was diagnosed with right focal hand dystonia, and MRgFUS left-sided Vo-thalamotomy for right hand dystonia was planned.

\section{Surgery and Postoperative Course}

With the patient under local anesthesia, a Cosman-Roberts-Wells stereotactic frame was fixed onto the skull after shaving the entire scalp. The stereotactic coordinates of the Vo nucleus were $1.5 \mathrm{~mm}$ posterior, $15 \mathrm{~mm}$ lateral, and 1.5 $\mathrm{mm}$ superior to the midcommissural point, which corresponded to the center of the coagulated lesion made by our radiofrequency Vo-thalamotomy. The procedure was performed with 3-T MRI (GE) and ExAblate Neuro systems (InSightec). During the treatment, an MRI-compatible guitar was used to assess the patient's dystonic symptoms (Fig. 1B). Repetitive low-power sonications for 10-20 seconds, inducing a temperature of $40^{\circ} \mathrm{C}$, were performed to evaluate the side effects. Subsequently, therapeutic sonications for 10-20 seconds, with gradual increases in acoustic power and energy, were applied (13 sonications; maximum temperature $63^{\circ} \mathrm{C}$ ). Immediately after therapeutic sonications, marked improvements were observed in the affected fingers while playing a guitar, without complications (Fig. 1C). Tubiana's MD scale (TMDS) was used to assess the neurological condition before and after MRgFUS Vo-thalamotomy as follows: score 1, subject plays several notes but stops because of blockage or lack of facility; score 2, subject plays short sequences without rapidity and with unsteady fingering; score 3 , subject plays easy pieces but cannot perform more technically challenging pieces; score 4, subject plays almost normally but avoids difficult passages because of fear or motor problems; score 5, subject returns to concert performances. ${ }^{17}$ Clinical follow-up with TMDS evaluations was performed at 0 and 1 week and 1,3,6, and 12 months after MRgFUS Vo-thalamotomy. Magnetic resonance imaging evaluations were performed immediately (Fig. 1D) and at 1 and 3 months (Fig. 1E) after MRgFUS Vo-thalamotomy. We had attempted to make a lesion with a volume of $300 \mathrm{~mm}^{3}$ on the T2-weighted MRI, and the volume of the created lesion was $348 \mathrm{~mm}^{3}$ (Fig. 1D). The TMDS scores before MRgFUS Vo-thalamotomy and at 0 and 1 week and 1,3, 6, and 12 months after MRgFUS Vothalamotomy were 1 (Video 1), 4, 4, 5, 5, 5, and 5 (Video 2), respectively.

VIDEO 1. Preoperative guitar play. Involuntary flexion of right second, third, and fourth fingers severely interfered in continuous fine movement in guitar play. Copyright Takaomi Taira. Published with permission. Click here to view.

VIDEO 2. Guitar play at 12 months after MRgFUS Vo-thalamotomy. Marked improvement of dystonic symptoms continued without any deficits. Copyright Takaomi Taira. Published with permission. Click here to view.

At the 12-month visit, the patient could play a classical guitar without any motor or sensory deficit.

\section{Discussion}

The present case showed that MRgFUS Vo-thalamotomy is effective for MD and is a less invasive procedure, which may be preferred not only for aged patients with an atrophic brain who are unsuitable for surgical procedures but also for young patients.

Gamma knife thalamotomy (GKT), which does not require craniotomy, is another less invasive procedure and is useful for treating tremor as well as focal hand dystonia. ${ }^{8,13}$ The long-term effect and safety of GKT have been reported. ${ }^{10,12,19}$ Permanent complications after GKT, including sensory and motor deficits, can occur in approximately $3 \%$ of cases and from unexpected lesion expansion. ${ }^{19}$ The biggest concern with GKT is the inability to deliver a test 
dose to confirm the optimal position for the lesion during the procedure. Recently, a long-term effect of FUS thalamotomy has been reported. ${ }^{2}$ Permanent neurological complications have included sensory (14\%) and gait (9\%) disturbance. ${ }^{3}$ However, there are limited reports of cases treated with FUS thalamotomy, and the reported follow-up periods are short compared to those for GKT. The longterm effect and safety of FUS thalamotomy have not been established. Magnetic resonance imaging-guided FUS Vothalamotomy produces lesions using thermal ablation and is expected to produce long-term improvement safely, as with radiofrequency thalamotomy? In the present case, the effects of Vo-thalamotomy persisted for 12 months without any complications. However, symptoms can recur after radiofrequency Vo-thalamotomy because of insufficient or incorrect thermocoagulation that does not cover the entire Vo nucleus of the thalamus. Even with the use of FUS thermocoagulation, symptom recurrence due to insufficient coagulation with a single sonication at peak energy has been reported, with an absence of coagulated lesions on MRI at 3 months. ${ }^{11}$ To maintain continuous therapeutic effects with permanent lesions, repetitive applications (4-5 times) of therapeutic sonications at the peak energy are required.

Although little attention has been paid to the surgical treatment of MD, several studies have shown the efficacy of Vo-thalamotomy not only for MD but also for other task-specific focal hand dystonias, such as writer's cramp, postapoplectic hemichorea, and paroxysmal kinesigenic dyskinesia. ${ }^{5,6,16}$ Although conservative treatments should be attempted first, in medically intractable cases, Vo-thalamotomy using FUS can be effective.

Additional studies with a larger sample size and longterm follow-up are needed to further elucidate the effectiveness of MRgFUS Vo-thalamotomy for MD.

\section{Acknowledgments}

We acknowledge Kaoru Shimizu and Masami Shirota of Intelligent Clinical Research and Innovation Center at Tokyo Women's Medical University for their invaluable assistance in the conduct of this study.

Takaomi Taira received research grants from Focused Ultrasound Foundation.

\section{References}

1. Altenmüller E, Jabusch HC: Focal dystonia in musicians: phenomenology, pathophysiology, triggering factors, and treatment. Med Probl Perform Art 25:3-9, 2010

2. Chang JW, Park CK, Lipsman N, Schwartz ML, Ghanouni $\mathrm{P}$, Henderson JM, et al: A prospective trial of magnetic resonance-guided focused ultrasound thalamotomy for essential tremor: results at the 2-year follow-up. Ann Neurol 83:107-114, 2018

3. Elias WJ, Lipsman N, Ondo WG, Ghanouni P, Kim YG, Lee W, et al: A randomized trial of focused ultrasound thalamotomy for essential tremor. N Engl J Med 375:730-739, 2016

4. Fahn S, Bressman SB, Marsden CD: Classification of dystonia. Adv Neurol 78:1-10, 1998

5. Goto S, Kunitoku N, Hamasaki T, Nishikawa S, Ushio Y: Abolition of postapoplectic hemichorea by Vo-complex thalamotomy: long-term follow-up study. Mov Disord 16:771-774, 2001
6. Horisawa S, Sumi M, Akagawa H, Kawamata T, Taira T: Thalamotomy for paroxysmal kinesigenic dyskinesias in a multiplex family. Eur J Neurol 24:e71-e72, 2017

7. Horisawa S, Taira T, Goto S, Ochiai T, Nakajima T: Longterm improvement of musician's dystonia after stereotactic ventro-oral thalamotomy. Ann Neurol 74:648-654, 2013

8. Horisawa S, Tamura N, Hayashi M, Matsuoka A, Hanada T, Kawamata T, et al: Gamma Knife ventro-oral thalamotomy for musician's dystonia. Mov Disord 32:89-90, 2017

9. Jabusch HC, Zschucke D, Schmidt A, Schuele S, Altenmüller E: Focal dystonia in musicians: treatment strategies and longterm outcome in 144 patients. Mov Disord 20:1623-1626, 2005

10. Lim SY, Hodaie M, Fallis M, Poon YY, Mazzella F, Moro E: Gamma knife thalamotomy for disabling tremor: a blinded evaluation. Arch Neurol 67:584-588, 2010

11. Magara A, Bühler R, Moser D, Kowalski M, Pourtehrani P, Jeanmonod D: First experience with MR-guided focused ultrasound in the treatment of Parkinson's disease. J Ther Ultrasound 2:11, 2014

12. Ohye C, Higuchi Y, Shibazaki T, Hashimoto T, Koyama T, Hirai T, et al: Gamma knife thalamotomy for Parkinson disease and essential tremor: a prospective multicenter study. Neurosurgery 70:526-536, 2012

13. Ohye C, Shibazaki T, Zhang J, Andou Y: Thalamic lesions produced by gamma thalamotomy for movement disorders. J Neurosurg 97 (5 Suppl):600-606, 2002

14. Schuele S, Jabusch HC, Lederman RJ, Altenmüller E: Botulinum toxin injections in the treatment of musician's dystonia. Neurology 64:341-343, 2005

15. Schuele S, Lederman RJ: Long-term outcome of focal dystonia in string instrumentalists. Mov Disord 19:43-48, 2004

16. Taira T, Hori T: Stereotactic ventrooralis thalamotomy for task-specific focal hand dystonia (writer's cramp). Stereotact Funct Neurosurg 80:88-91, 2003

17. Tubiana R: Musician's focal dystonia. Hand Clin 19:303308, vii, 2003

18. van Vugt FT, Boullet L, Jabusch HC, Altenmüller E: Musician's dystonia in pianists: long-term evaluation of retraining and other therapies. Parkinsonism Relat Disord 20:8-12, 2014

19. Young RF, Li F, Vermeulen S, Meier R: Gamma Knife thalamotomy for treatment of essential tremor: long-term results. J Neurosurg 112:1311-1317, 2010

\section{Disclosures}

Dr. Taira has received lecture fees from Insightec Ltd.

\section{Author Contributions}

Conception and design: Taira, Yamaguchi. Acquisition of data: all authors. Analysis and interpretation of data: Taira. Drafting the article: Horisawa. Critically revising the article: Taira, Horisawa. Approved the final version of the manuscript on behalf of all authors: Taira. Study supervision: Taira, Yamaguchi.

\section{Supplemental Information \\ Videos \\ Video 1. https://vimeo.com/277439192. \\ Video 2. https://vimeo.com/277439212.}

\section{Correspondence}

Takaomi Taira: Tokyo Women's Medical University, Tokyo, Japan.ttaira@twmu.ac.jp. 